\title{
NOTES
}

\section{XPS Study of Boundary Phase Structure between Stereoregular Poly(methyl methacrylate) and Polyamide Substrate}

\author{
Minekazu Kodama and Kazuo Kuramoto \\ Materials \& Electronic Devices Laboratory, Mitsubishi Electric Corporation, \\ 1-1, Tsukaguchi-Honmachi 8-chome, Amagasaki, Hyogo 661, Japan \\ (Received December 17, 1987)

\begin{abstract}
KEY WORDS X-Ray Photoelectron Spectroscopy / Boundary Phase Structure / Stereoregular Poly(methyl methacrylate) / Polyamide Substrate / Molecular Conformation / Substrate-Polymer Interaction /
\end{abstract}

It was reported in the previous paper ${ }^{1}$ from the study of band frequency shift in Fouriertransform infrared spectroscopy that an interaction arose between the amide group in Kevlar-fiber ${ }^{\circledR}$ and the carbonyl group of poly(methyl methacrylate) (PMMA) in Kevlar-fiber reinforced PMMA. The interaction between the fiber and matrix and the boundary phase structure formed in the vicinity of the reinforcement are expected to vary depending on the tacticities of PMMA.

The purpose of this work is to elucidate the boundary phase structure between nylon$6(\mathrm{Ny})$ substrate and PMMA in relation to the difference in tacticity of PMMA by X-ray photoelectron spectroscopy (XPS). Ny was shown $^{2}$ to be usable as a model compound for Kevlar-fiber from the Fourier-transform infrared spectroscopy.

The PMMA samples used in this work were obtained from Polymer Laboratories Inc. and used as received. The characterizing data of these polymers from the supplier are shown in Table I. The Ny plate of $1 \mathrm{~mm}$ thick was obtained by compression molding Novamide 1010 (nylon-6 obtained from Mitsubishi Chemical Industries, Co.). The cleaned $\mathrm{Ny}$ plate was dip-coated with a $2 \%$ PMMA solution in tetrahydrofuran at a thickness of $c a$.
$0.5 \mathrm{~mm}$ by repeated coating. After removal of the solvent, the film of PMMA formed on $\mathrm{Ny}$ substrate was carefully separated after 1 week from the substrate so as not to damage the film surface. By leaving the coated specimen for 1 week in the air, the film became easily separated from the substrate. XPS spectra were measured for the separated films on the surfaces exposed to the air (air-facing side) and Ny substrate ( $\mathrm{Ny}$-facing side).

XPS spectra were obtained with a VG HB 50A scanning electron microscope equipped with XPS option. An aluminum anode X-ray source producing AlKa X-ray at $1486.6 \mathrm{eV}$ operating at $12 \mathrm{kV}$ and $20 \mathrm{~mA}$ was used. The degree of vacuum of the instrument was maintained at least at $1 \times 10^{-8} \mathrm{mbar}$ for all measurements. The data were analyzed on the high resolution spectra of the $C_{1 s}$ region. The overlapping peaks in the $\mathrm{C}_{1 \mathrm{~s}}$ spectra were

Table I. Characterization data for PMMA

\begin{tabular}{|c|c|c|c|c|c|}
\hline & \multirow{2}{*}{$\bar{M}_{w} \times 10^{-3}$} & \multirow{2}{*}{$\bar{M}_{w} / \bar{M}_{n}$} & \multicolumn{3}{|c|}{ Tacticity, $\%$ Triads } \\
\hline & & & $\mathrm{mm}$ & $m r$ & $r r$ \\
\hline Isotactic & 178 & 25.6 & 90 & 6 & 4 \\
\hline Atactic & 140 & 1.1 & 7 & 40 & 53 \\
\hline Syndiotactic & 262 & 2.6 & 6 & 18 & 76 \\
\hline
\end{tabular}


M. Kodama and K. Kuramoto
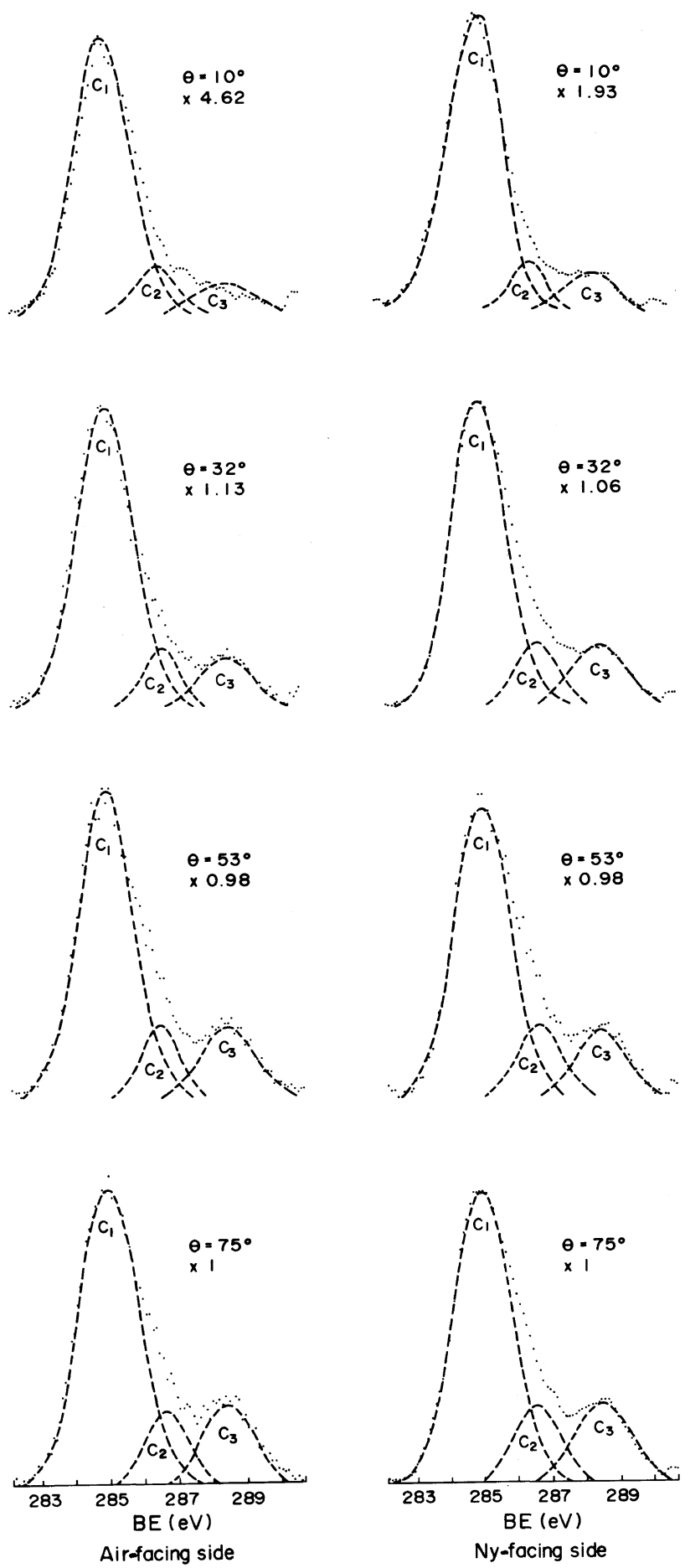

Figure 1. Take-off angle $(\theta)$ dependence of the observed $\mathrm{C}_{\mathrm{ls}}$ spectra $(\cdot)$ and resolved components $(-)$ for isotactic PMMA at the air-facing and Ny-facing sides. 
resolved into their individual components after background subtraction, assuming a Gaussian type symmetric function with respect to the photoelectron intensity versus binding energy. ${ }^{2}$ Charge correction in the binding energy scale was made by setting the peak for hydrocarbon component at $285.0 \mathrm{eV}$ in binding energy. Angular-dependent XPS measurements were carried out by tilting the surface of sample so that the effective sampling depth would decrease with decreasing take-off angle of photoelectron $(\theta)$, which is the angle between the surface of the sample and electron analyser. $^{2}$

In Figure 1, the observed $\mathrm{C}_{1 \mathrm{~s}}$ spectra (dotted curve) and resolved components (broken curve) are shown for both the air-facing and the Ny-facing sides at various take-off angles for isotactic PMMA. The resolved components $\mathrm{C}_{1}, \mathrm{C}_{2}$, and $\mathrm{C}_{3}$ can be assigned to $\underline{\mathrm{C}} \mathrm{Hx}, \underline{\mathrm{C}}-\mathrm{O}$, and $\mathrm{O}-\mathrm{C}=\mathrm{O}$, and center at 285.0, 286.8 and $288.7 \mathrm{eV}$, respectively. ${ }^{3,4}$ For atactic and syndiotactic PMMA, the resolved components also center at the same binding energy as for isotactic PMMA.

The boundary phase structure was probed by comparing the XPS spectra between the Ny-facing and air-facing sides.

The relative peak areas of $\mathrm{C}_{1}, \mathrm{C}_{2}$, and $\mathrm{C}_{3}$ components were obtained, and the sum of the relative peak areas of $\mathrm{C}_{2}$ and $\mathrm{C}_{3}$ components $\left[\left(\mathrm{C}_{2}+\mathrm{C}_{3}\right)\right.$ ratio] is plotted against the take-off angle $(\sin \theta)$ in Figure 2. $\left(\mathrm{C}_{2}+\mathrm{C}_{3}\right)$ ratios are somewhat smaller compared with the values anticipated from the chemical structure $(40 \%)$ in both the air-facing $(\mathrm{O})$ and $\mathrm{Ny}$-facing sides (O) irrespective of tacticity. This is probably due to the increase in relative peak area of $C_{1}$ component on account of the hydrocarbon contamination layer deposited on the sample during measurement. ${ }^{5}$ The $\left(\mathrm{C}_{2}+\mathrm{C}_{3}\right)$ ratio at the air-facing side decreases with decreasing $\sin \theta$ irrespective of the tacticity of the sample. Hook et al. ${ }^{6}$ report that ratios of $\mathrm{C}_{2}$ and $\mathrm{C}_{3}$ components do not vary depending on sampling depth. Our data are contradictory to their

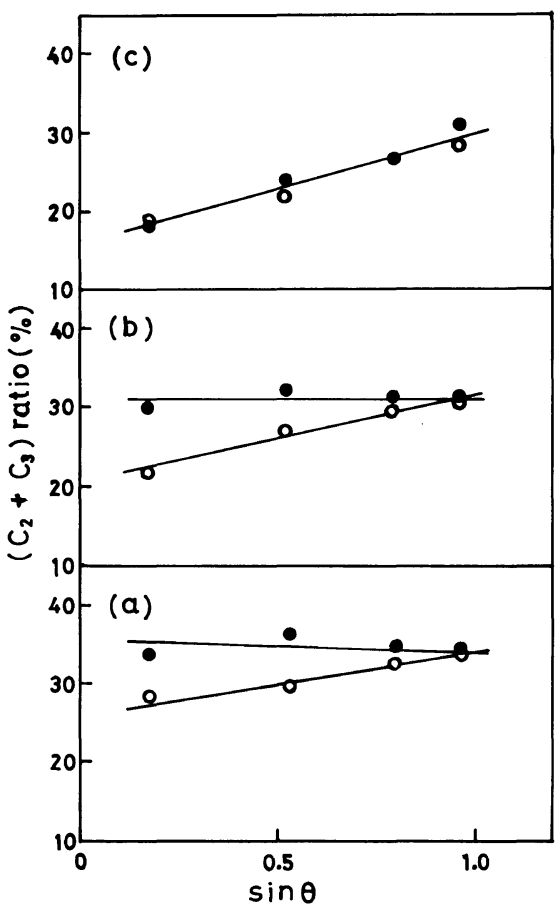

Figure 2. Take-off angle $(\theta)$ dependence of the sum of relative peak area of $C_{2}$ and $C_{3}$ components $\left[\left(C_{2}+C_{3}\right)\right.$ ratio] for (a) atactic PMMA, (b) syndiotactic PMMA, and (c) isotactic PMMA at the air-facing side $(O)$ and Ny-facing side (O).

results in appearance. As the effect of hydrocarbon contamination, however, possibly becomes larger with decrease of sampling depth, ${ }^{5}$ detailed discussion cannot be made of the dependence of $\left(C_{2}+C_{3}\right)$ ratio on $\sin \theta$ by this experiment alone. As a consequence, it may be said that the remarkable differences in $\left(\mathrm{C}_{2}+\mathrm{C}_{3}\right)$ ratio versus $\sin \theta$ relation cannot be seen among samples at the air-facing side.

At the $\mathrm{Ny}$-facing side of atactic and syndiotactic samples, the slope of the $\left(\mathrm{C}_{2}+\mathrm{C}_{3}\right)$ ratio versus $\sin \theta$ relation decreases as a result of the increase in the $\left(\mathrm{C}_{2}+\mathrm{C}_{3}\right)$ ratio at the lower take-off angle, as compared with the case of the air-facing side. On the contrary, $\left(\mathrm{C}_{2}+\mathrm{C}_{3}\right)$ ratio versus $\sin \theta$ relation can hardly be distinguished between the $\mathrm{Ny}$-facing and air-facing sides in the case of the isotactic sample. That is, the carboxylic group is enriched in the 
vicinity of the $\mathrm{Ny}$ surface, probably on account of the interaction between it and the carboxylic group, in both cases of atactic and syndiotactic PMMA. The enrichment of carboxylic group, however, cannot be seen in the case of isotactic PMMA. In other words, susceptibility to the influence of the $\mathrm{Ny}$ substrate is substantially small for isotactic PMMA compared with atactic and syndiotactic PMMA.

The characteristic ratio $\left\langle r^{2}\right\rangle_{0} / n l^{2}$, in which $\left\langle r^{2}\right\rangle_{0}, n$ and $l$ are the unperturbed mean square end-to-end distance, the number of skeletal bonds and the average length of each bond, is used as a parameter representing the overall molecular conformation or the degree of constraint on the rotation of the molecular chain. The characteristic ratios for atactic, syndiotactic and isotactic PMMA were obtained as 7.5, 7.3 , and 10.3 from the solution properties in tetrahydrofuran $^{7}$ and as 7.9, 9.2, and 10.7, from the bulk properties, ${ }^{8}$ respectively. These data show that isotactic PMMA is more extended in the unperturbed state or more restricts the rotation of molecular chain compared with atactic and syndiotactic PMMA.

The carboxylic groups in isotactic PMMA may have lesser chance to come close to the $\mathrm{Ny}$ surface in the course of sample preparation on account of the extended chain conformation or the larger degree of constraint on the rotation of the molecular chain. On the contrary, the carboxylic group in atactic and syndiotactic PMMA may be able to come close to the $\mathrm{Ny}$ surface on account of the somewhat compact conformation or lesser degree of constraint on the rotation of molecular chain. These situations may contribute to differences in the boundary phase structure between stereoregular PMMAs and the Ny substrate.

\section{REFERENCES}

1. M. Kodama and I. Karino, J. Appl. Polym. Sci., 32, 5057 (1986).

2. M. Kodama, K. Kuramoto, and I. Karino, J. Appl. Polym. Sci., 33, 1889 (1987).

3. T. J. Hook, R. L. Schmitt, J. A. Gardella, Jr., L. Salvati, Jr., and R. L. Chin, Anal. Chem., 58, 1285 (1986).

4. S. Suzuki, Y. Inoue, I. Ando, R. Chûjô, K. Seto, and K. Hoshino, Polym. J., 7, 8 (1975).

5. D. H. K. Pan and W. M. Prest, Jr., J. Appl. Phys., 58, 2861 (1985).

6. T. J. Hook, J. A. Gardella, Jr., and L. Salvati, Jr., J. Mater. Res., 2, 117 (1987).

7. R. Jenkins and R. S. Porter, Polymer, 23, 105 (1982).

8. J. M. O'Reilly, D. M. Teegarden, and G. D. Wignall, Macromolecules, 18, 2747 (1985). 